\title{
Analysing EEG signals for mathematical computations
}

\author{
Martin Strmiska ${ }^{1, *}$, Zuzana Koudelkova ${ }^{2}$ \\ ${ }^{1}$ Tomas Bata University in Zlin, Faculty of Applied Informatics, Department of Automation and Control Engineering, Nad Stranemi \\ 4511, 76005 Zlin, Czech Republic \\ ${ }^{2}$ Tomas Bata University in Zlin, Faculty of Applied Informatics, Department of Informatics and Artificial Intelligence, Nad Stranemi \\ 4511, 76005 Zlin, Czech Republic
}

\begin{abstract}
Brain computer interface (BCI) is a device that allows us to scan brainwaves. Achieved signals can be processed using a computer and the analyzed brain activity can be than monitored. In this paper, the use of the non-invasive brain scanning method applied on person at solving a system of equations is described. This solving the system of equations was obtained by two mathematical methods. The measurement was performed for solving equations by Gaussian elimination and by substitution methods separately. The results of the measurements were visualized by graphing the brain activity. The aim of the work is to determine the more practical method of those two.
\end{abstract}

\section{Introduction}

The EEG curve (Electroencephalogram) is an entry of the change in time of electric capability (EEG signal) known as brain activity. The signal is different for everybody and it varies with age, gender, alertness and other factors. The character of the signal is mostly similar for same kind of human activities. That makes the signal worthy to evaluate. More information about the EEG curve can be found here. [1]-[7]

There are three main methods to capture the signal. It can be captured by an invasive, partially invasive and non-invasive method. Invasive method requires neurosurgical procedure involving opening the scull and implanting the electrodes right to the grey cerebral cortex. The method guarantees the highest quality of signal received. However, there is a risk of permanent brain damage connected with the invasive method. Partially invasive method requires electrodes to be connected inside of the skull, but also outside the brain. The signal has higher quality than the signal captured by non-invasive method and the risk of cerebral tissue's damage is lower. Non-invasive method is the least risky of those three. The signal is captured from the surface of the head. These methods are described here [2]. [1]-[7]

For this work, we have used the device BCI Emotive $E P O C+$ that captures brain signal using the non-invasive method by measuring brain waves from the skull surface.

In our case, the signal is measured and processed directly by an app called EmoBrainMap. This application allows to process and display the vary of the brain signal: alpha, beta, delta and theta waves.

This article deals with measuring the brain wave signal during the process of solving the system of equations by two methods (Gaussian elimination and substitution method) using the version of the app EmoBrainMap. [1]-[7]

The first part of the article defines the system of equations and methods used to solve it (Gaussian elimination method and substitution method).

The chapter explaining the basic brain hemisphere activity follows. Brain waves, such as alpha, delta, theta and especially beta waves (important for mathematical, logical and analytical thinking) are described further.

After that, we move on to the experimental part with all the results. The last part of this article is the conclusion chapter, where we determine which method is more practical on the base of results from brain signal measurement.

\section{System of linear equations}

Let's have a system (1) according to rules in [8]:

$$
\begin{gathered}
a_{11} x_{1}+a_{12} x_{2}+\cdots+a_{1 n} x_{n}=b_{1} \\
a_{21} x_{1}+a_{22} x_{2}+\cdots+a_{2 n} x_{n}=b_{2} \\
\vdots \\
a_{m 1} x_{1}+a_{m 2} x_{2}+\cdots+a_{m n} x_{n}=b_{n}
\end{gathered}
$$

where $a_{11} \ldots a_{m n} \in \mathbb{R}$ and $b_{1} \ldots b_{n} \in \mathbb{R}$. Then we call the upper schema the system of linear equations. Particular application of this system can be seen e.g. in [9].

\subsection{Gaussian elimination method}

The Gaussian elimination method consists of transforming the original system of linear equations to

* Corresponding author: $\underline{\text { strmiska@,utb.cz }}$ 
the new simplified system of the linear equations. These two systems are equivalent. Row reduction makes use of elementary row operations (we are working with numbers instead of variables). This method is further described here. [6]

\subsection{Substitution method}

The substitution method works with both coefficients and variables. With the help of the operation called substitution we convert the system of $n$ equations to the system of $n-1$ equations and process is repeated until we find a root. Then with the use of substitution the rest of the roots are calculated. [6]

\section{Central Nervous System}

The nervous system consists of central nervous system (CNS) and peripheral nervous system (PNS). The parts of the CNS are brain and spinal cord. Nerves and ganglia outside the brain together with the spinal cord form PNS. Vital body functions (heartbeat, breathing), muscle movement and cognitive functions as thinking, speech, memory retention and emotion processing are managed by the brain itself.

It is commonly divided to two hemispheres: left and right hemisphere. Each of those serves to a different purpose but they are actively collaborating. People usually have one hemisphere dominant and other one complementary.

The right hemisphere helps with factual and logical thinking, language, exact science, math, pragmatic and analytical thinking, organizing. The left hemisphere relates to emotions, imagination, art perception, sport and synthetic thinking. [7]

\subsection{Brainwaves}

Electromagnetic field around the brain is composed by brainwaves. It is created by electrical activity of neurons. The most frequently mentioned are: Alpha, Beta, Gamma, Delta and Theta waves. These are all active in certain moments in human life. Their frequency is measured in Hertz (Hz). [7]

- Gamma waves $(40-100 \mathrm{~Hz})$ play important role in thinking and memory. They are emitted when the brain is exposed to difficult tasks.

- Beta waves $(12-40 \mathrm{~Hz})$ dominate the field in conscious state. Higher frequencies signal irritation, anger, nervousness or fear. Lower frequencies indicate tiredness or sleepiness. Their activity is higher when the brain applies logical and analytical thinking to solve a task or problem. This part of brainwave spectrum is the key examination factor for this work.

- Alpha waves $(8-12 \mathrm{~Hz})$ are dominant in the state of release and rest, when we are not bombarded by information or actions requiring our immediate attention.
- Delta waves $(1-4 \mathrm{~Hz})$ show up in a state of deep meditation, deep sleep or unconsciousness. Their presence implies that the body is in a process of deep regeneration.

- Theta waves $(4-8 \mathrm{~Hz})$ are active during deep relaxation, meditation and sleep. The long-term memory is being stimulated and nonobvious solutions come up in this state. [7]

\section{Brain Computer Interface}

The connection of BCI device to the brain and computer serves as a signal transfer line. Brain signals are captured by the device and sent to the computer to for analysis. The computer receives the EEG signals and process it via software tool, then displays the analysed signal in a visualised form to the researcher.

The picture bellow shows the use of the device. First the electrodes are lubricated and then scanning helmet is put on head. The signal captured by electrodes is amplified, transformed to the digital form and sent to the computer wirelessly.

The measurement process itself does not require any special collaboration from a subject, except from executing the given task. $[2,5]$

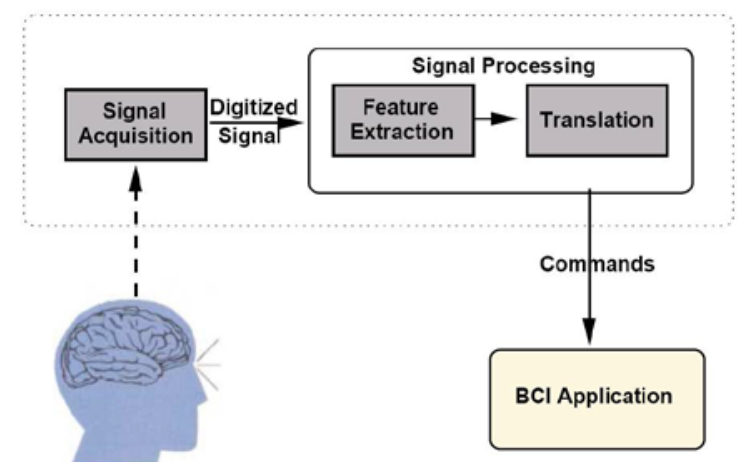

Fig. 1. Diagram of BCI system.

\subsection{Emotiv EPOC+}

Emotiv EPOC+ device is intended for scalable and contextual human brain research. The kit comes with a set of helmet and proprietary USB receiver. We can observe the brain activity during emotional expressions and thinking processes on a colourful real-time animation. [4]

The 16 scalp sensors are bit tough to attach correctly but work seamlessly. The software will indicate the correct attachment of each sensor by picturing the sensor green on output visualisation. [4] 


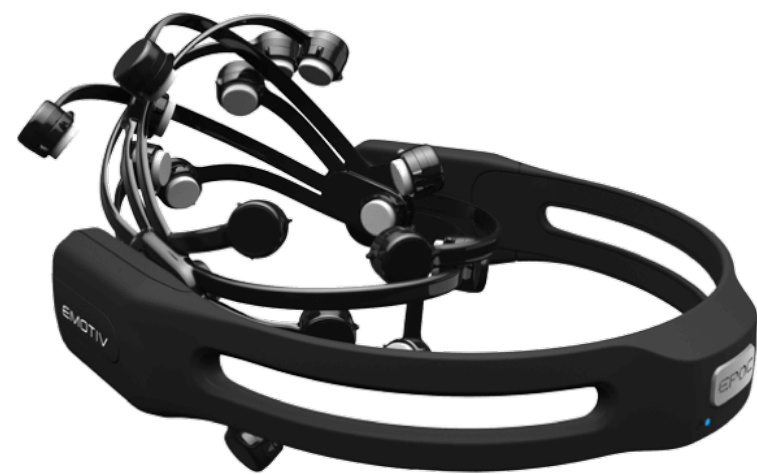

Fig. 2. Emotiv EPOC+.

\section{Experimental Part}

\subsection{Aims of the experiment}

The main goal of the experiment was to compare two mathematical methods for solving Systems of linear equations (Gaussian elimination and substitution method) and determine which one is easier to use.

The authors assume, that the easier solution comes with the use of Gaussian elimination method, because it operates mostly with numbers. The solving process is harder on the contrary with substitution method because the solver must think which variables should be substituted with expression and manipulate with fractions, which is usually more complicated operation.

\subsection{Description of the experiment}

There were total of five people participating in this experiment. Each subject was given the same task and same set of instructions.

The instruction was to solve the system of linear equations first by Gaussian elimination and then by substitution method. The given linear system (2):

$$
\begin{aligned}
& 4 x_{1}+3 x_{2}-9 x_{3}=9 \\
& 2 x_{1}+5 x_{2}-8 x_{3}=8 \\
& x_{1}+8 x_{2}-7 x_{3}=12
\end{aligned}
$$

had just one correct solution: $K=\{[3,2,1]\}$.

\subsection{Results of experiment}

All subjects showed higher brain activity when solving the system by substitution, in contrast with Gaussian elimination where activity was lower. Notice the brain activity during solving the system of equation by Gaussian elimination on the picture (Figure 3). Mostly right brain hemisphere was dominant (not responsible for mathematics and logic but for simple operations).

The next picture (Figure 4) shows a subject's brain activity visualization when solving the system by substitution method. The higher brain activity in left hemisphere (the one which is responsible for logical and mathematical thinking) is noticeable.

The conclusion is that the Gaussian elimination method for solving linear equations is easier for a human brain to process than the other method.

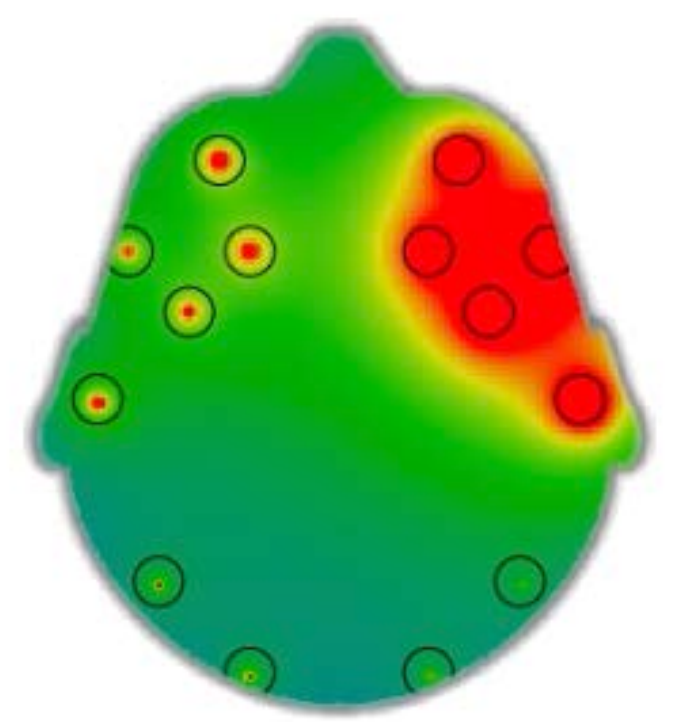

Fig. 3. Brain activity during the use of Gaussian elimination.

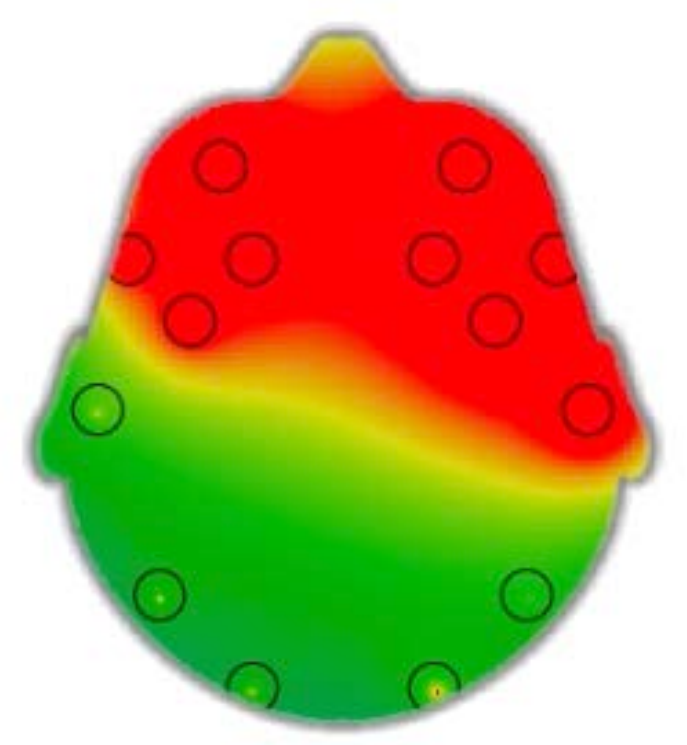

Fig. 4. Brain activity during the use of substitution method.

\section{Conclusion}

The advantage of BIC is its multipurpose usage for scientific and recreational purposes (for example to play games using thinking) as well. The scientific usage includes: brain activity analysis (evaluating the complexity level of certain activities), disease detection, mind-controlling the devices, improving concentration (training the brain to manage thoughts efficiently).

Our work was focused on finding out which mathematical method for solving systems of linear 
equations from two handpicked methods is easier to use. The practical use of the knowledge is, that we can use it identify harder tasks and replace them with easier equivalents.

Our university collaborates with University Hospital Ostrava, which tries to implement BCI use to improve the rehabilitation process. The tasks which seem to be too complicated for patients (measured by scanning EEG signals) are replaced by more pleasant tasks for a patient. Thanks to the BCI systems the neuro-deficient patient has the possibility to actively participate in rehabilitation process with help of mind self-regulation and concentration improving techniques.

The university plans to buy full paid version of the software, when research results show greater success in the future. The paid version includes processing the EEG signal using statistics or neural networks.

The outcome we get from the research will be useful for developing software that will help doctors diagnose brain related diseases and anomalies more effectively and accurately.

\section{Acknowledgement}

This work was supported by Internal Grant Agency of Tomas Bata University in Zlín under the project No. IGA/FAI/2019/005.

\section{References}

1. S. S. Mader, M. Windelspecht, Human biology, 12th ed., (New York, NY: McGraw-Hill, 2012), ISBN 0073525464

2. H.S. Anupama, N.K. Cauvery, G.M. Lingaraju, International Journal of Advances in Engineering \& Technology, 3, 739-745 (2012)

3. S. Siuly, Y. Li, Y. Zhang, EEG signal analysis and classification, (New York, NY: Springer Berlin Heidelberg, 2017), ISBN 9783319476520.

4. EMOTIV EPOC+ - 14 Channel Wireless EEG Headset [online], in: https://www.emotiv.com/epoc/

5. B. He, Neural Engineering Second Edition, (New York, NY: Springer Berlin Heidelberg, 2013), ISBN 9781489978875

6. Atkinson, Kendall A. (1989), An Introduction to Numerical Analysis (2nd ed.), New York: John Wiley \& Sons, ISBN 978-0471624899.

7. 5 types Of Brain Waves Frequencies [online], in: https://mentalhealthdaily.com/2014/04/15/5-typesof-brain-waves-frequencies-gamma-beta-alphatheta-delta/

8. A.M. Robert. Linear Algebra: examples and applications, (Hackensack, 2005), ISBN 981-256499-3

9. T. Barot, Cybernetics and Algorithms in Intelligent Systems, AISC, 765, 53-62, Springer (2019) 